\title{
Valoración participativa de servicios ecosistémicos prestados por el humedal Ramsar de Moyúa, Ciudad Darío
}

\section{Participatory assessment of ecosystemic services provided by the Ramsar wetland of Moyúa, Ciudad Darío}

\section{Francisco Javier Chavarría Aráuz ${ }^{1}$, Anielka K. Chavarría López ${ }^{2}$, Josseling V. González Obregón ${ }^{2}$}

${ }^{1} \mathrm{PhD}$. en Desarrollo Rural y MSc. en Medio Ambiente y RRNN, Docente Investigador UNAN Managua-FAREM Matagalpa; ORCID: https://orcid.org/0000-0003-1933-6260 / fcha29@yahoo.com

2Ingeniera agrónomo, graduadas en UNAN Managua/FAREM Matagalpa / anichalo04@yahoo.com

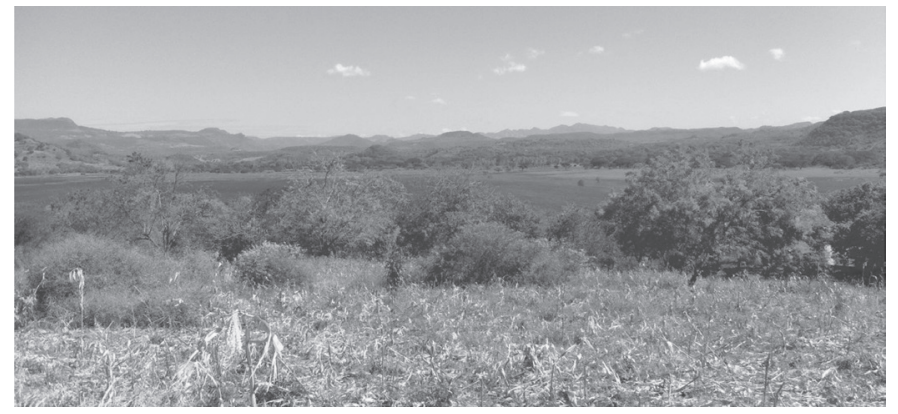

\begin{abstract}
RESUMEN
El estudio de servicios eco sistémicos y sobre todo los obtenidos de un humedal, revierte gran interés para poder justificar la importancia de estos sitios por su valor económico, social y ambiental. El objetivo principal del estudio fue identificar y valorar los servicios ecosistémicos prestados por el sitio Ramsar a los pobladores de dicho lugar. El método utilizado es Valoración contingente. La información se recopiló mediante grupos focales, lista de chequeo de servicios ecosistémicos y Escala Likert. El universo de estudio es igual a la muestra. Los resultados obtenidos concuerdan con la hipótesis planteada ya que los pobladores tienen conciencia del valor que poseen los servicios ecosistémicos prestados por el humedal. El $100 \%$ de los pobladores que participaron en el presente estudio, manifestaron saber el valor que tienen los servicios ecosistémicos que presta el sitio Ramsar, los cuales se están viendo afectados por el cambio climático, siendo necesario implementar medidas de conservación del agua, suelos y bosques. Se considera necesaria la participación activa de los pobladores, mayor involucramiento de la alcaldía de ciudad Darío, Ministerio de Ambiente y Recursos Naturales, Ministerio de Agricultura y Ganadería y Universidad Nacional Autónoma de Nicaragua-Managua, a fin de lograr la conservación y restauración de las funciones de los ecosistemas.

Palabras clave: servicios de aprovisionamiento, contingente, restauración, conservación, prácticas.
\end{abstract}

\begin{abstract}
The study of eco-systemic services and especially those obtained from humidity, reverts great interest to be able to justify the importance of these sites for their economic, social and environmental value. The main objective of the study is to identify and assess ecosystem services provided by the Ramsar site to the inhabitants of that place. The method used is contingent valuation. The information was collected through focus groups, Checklist of ecosystem services and Likert Scale. The universe of study is equal to the sample. The results obtained are consistent with the hypothesis raised as the residents are aware of the value of the ecosystem services provided by the wetland. $100 \%$ of the residents who participated in this study, said they know the value of the ecosystem services provided by the Ramsar, which are being affected by climate change, being necessary to implement water, soil and forest conservation measures. It is considered necessary the active participation of the inhabitants, greater involvement of the City Hall of ciudad Darío, Ministerio de Ambiente y Recursos Naturales, Ministerio de Agricultura y Ganadería y Universidad Nacional Autónoma de Nicaragua-Managua, in order to achieve the conservation and restoration of ecosystem functions.
\end{abstract}

Keywords: Provisioning services, contingent, restoration, conservation, practices.
Recibido: 19 de febrero del 2019

Aceptado: 20 de abril del 2020

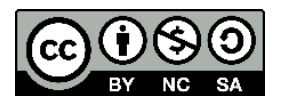

Los artículos de la revista La Calera de la Universidad Nacional Agraria, Nicaragua, se comparten bajo términos de la licencia Creative Commons: Reconocimiento, No Comercial, Compartir Igual. Las autorizaciones adicionales a las aquí delimitadas se pueden obtener en el correo edgardo.jimenez@ci.una.edu.ni 


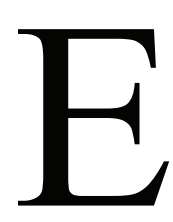

s muy compleja la situación de degradación de los principales componentes del capital natural de los ecosistemas que forman parte del humedal PlayitasMoyúa-Tecomapa, ubicado en el municipio de Ciudad Darío, Matagalpa, Nicaragua; declarado como sitio Ramsar por su importancia social, económica $\mathrm{y}$ ambiental. Al igual que en otros paisajes y casos, las labores de explotación irracional de los recursos naturales y especialmente los bosques, se ha dado la fragmentación y destrucción de los hábitats, afectando a numerosas especies de fauna (Santos y Tellería, 2006).

La transformación de los paisajes como producto de la inadecuada planificación y gestión de los espacios naturales, afecta seriamente a todos losprocesos ecológicos y por ende las funciones y la prestación de servicios ecosistémicos, que son de vital importancia para la sobrevivencia y el bienestar humano y de las especies con que se relaciona.

En el estudio se consideraron aspectos sociales, ecológicos, económicos, culturales y de paisaje. Con los resultados del estudio que se presenta de manera sucinta a continuación, se pretende contribuir al diseño y aplicación de políticas para la adecuada gestión de los recursos hídricos, los suelos y la biodiversidad. El conocimiento de las funciones y los servicios ecosistémicos que proveen los humedales como el de Laguna de Moyúa, es de vital importancia para en primer lugar lograr la concienciación de la población y las autoridades locales y en base a ello impulsar el manejo integrado de los ecosistemas y sobre todo de los recursos hídricos.

El tipo de servicio proporcionado por un área geográfica determinada, contribuye a lograr un mejor control sobre las metas de protección ecosistémica a escala general, y optimizar el manejo de la oferta, demanda y flujo de servicios ecosistémicos a un nivel de mayor precisión. Por otro lado, la evaluación de los servicios ecosistémicos se convierte en una poderosa herramienta para los tomadores de decisiones, al momento de planificar programas, proyectos y simple acciones para la preservación, recuperación y mejoramiento de los ecosistemas. Por lo antes expuesto, el presente trabajo de investigación tiene por objetivo contribuir a concienciar sobre la importancia de identificar, caracterizar y valorar, con una visión integradora de la interface naturaleza-sociedad, los bienes y servicios ecosistémicos de los agroecosistemas existentes en el humedal Moyúa, así como su contribución para el desarrollo sustentable de la zona de estudio.

\section{MATERIALES Y MÉTODOS}

Descripción de la zona de estudio. El estudio se llevó a cabo en parcelas ubicadas en la comunidad Los Morenos, en el sitio Ramsar Playitas-Moyúa-Tecomapa, ubicada a $70 \mathrm{~km}$ de Managua. Este sector pertenece al municipio de Ciudad Darío, Departamento de Matagalpa (Aguilera, 2010). La investigación es de tipo descriptiva, con un enfoque mixto (cualicuantitativa), de corte transversal por considerarse un periodo de tiempo determinado. Por su parte cualitativa, tiene un enfoque de Investigación-Acción-Participativa. Ya que se pretendió identificar el problema, se buscaron las causas que explican desde la óptica de los comunitarios y formular las propuestas que logran incidir en la búsqueda de solución al problema (o problemas). Las investigaciones descriptivas (como la presente) parten del hecho de que hay una cierta realidad (o sector del mundo) que resulta insuficientemente conocida y, al mismo tiempo, relevante e interesante para ciertos desarrollos. En el caso de los servicios ecosistémicos, ha sido poco abordado a nivel nacional. De hecho, no se encontró información local sobre esta temática.

Población y muestra. La población sujeta de estudio, está integrada por todos los productores del Sector Los Morenos, compuesta de 12 productores, que por ser una cantidad pequeña se decidió considerarlos en un cien por ciento como muestra. En la presente investigación, se aplicaron como principales técnicas; el grupo focal, lista de chequeos y escala Likert con valoración del 1 al 8. En los grupos focales se presentó lista de chequeo sobre los servicios ecosistémicos que los participantes del estudio logran percibir que reciben de los diferentes componentes del ecosistema. Estos resultados se compararon posteriormente con la tabla de servicios ecosistémicos de la Evaluación de los Ecosistemas del Milenio (MEA, 2005). Se llevaron a cabo sesiones de trabajo con los pobladores para la presentación de la información resultante y obtener su opinión y aportes, para finalmente lograr una versión mejorada de la propuesta de planificación y gestión de las unidades de producción y su entorno.

Para estimar consumo de agua para ganado bovino, se aplicó ecuación de Murphy, considerando su producción de leche, el consumo de sales minerales (cloruro de sodio) y la temperatura ambiente media al día (bajo sombra y a pleno sol). La ecuación es la siguiente: Consumo $(\mathrm{L} /$ día/UAM $)=$ $15.99+(1.58 * \mathrm{IMS})+(0.9 *$ P.L. $)+(0.05 * \mathrm{C} . \mathrm{Na})+\left(1.2 * \mathrm{~T}^{\mathrm{a}}\right)$.

Procesamiento de la información. Los datos recopilados en los grupos focales por medio de lista de chequeo y la escala Likert se plasmaron en hoja de cálculo de Excel. Con ayuda del software SPSS, se logró encontrar contrastes de asociaciones entre categorías de las variables. Para ello se utilizaron tablas de contingencia (Balzarini et al., 2008).

\section{RESULTADOS Y DISCUSIÓN}

Resulta difícil abordar de forma aislada cada una de las funciones y servicios que brindan los ecosistemas y más tratándose de sistemas tan frágiles, pero a la vez tan importantes como lo son los humedales de agua dulce. Por ello al momento de presentar y analizar los resultados de la presente investigación, se hace evidente la interrelación entre ellos. 
Existencia de servicios ecosistémicos. La identificación de los servicios ecosistémicos se realizó utilizando lista de chequeo mejorada, previamente diseñadas en base a información contenida en trabajos de la Iniciativa Evaluación de Ecosistemas del Milenio (MEA, 2005).

Los servicios ecosistémicos identificados se clasifican en cuatro grupos, subdivididos en indicadores. Los servicios ecosistémicos de aprovisionamiento, de regulación, cultural y de soporte. Los comunitarios generalmente se fijan en los servicios de aprovisionamiento, principalmente por los alimentos, agua de consumo, leña, medicina, fibras, tintes, bioquímicos. En los de regulación lograron identificar el papel en el clima, control de plagas y enfermedades, polinización, saneamiento de agua y contaminantes. Los servicios culturales que más sobresalen son el valor espiritual, valor recreativo, turístico, inspirativo, educativo, herencia cultural e identidad.

Al realizar análisis de correlaciones entre las variables: a) Valoración de la existencia de la laguna y sus servicios ecosistémicos y b) el bienestar de las familias que habitan en las cercanías. Para el análisis se aplicó estadísticos descriptivos como Tabla de Contingencia. El coeficiente de Pearson cuya significación asintótica resultó ser de 0.069 , indica que la población considera que su bienestar depende de la existencia de la laguna y los servicios ecosistémicos que eso representa(Bortz,LienertyBoehnke, 1990).Esdecir,serechaza que exista independencia entre las variables contrastadas.

Cuadro 1. Resultados de Chi cuadrado tabla de contingencia

\begin{tabular}{lccccc}
\hline \multicolumn{1}{c}{ Pruebas } & Valor & gl & $\begin{array}{c}\text { Sig. asintótica } \\
\text { (bilateral) }\end{array}$ & $\begin{array}{c}\text { Sig. exacta } \\
\text { (bilateral) }\end{array}$ & $\begin{array}{c}\text { Sig. exacta } \\
\text { (unilateral) }\end{array}$ \\
\hline Chi-cuadrado de Pearson & $0.069^{\mathrm{a}}$ & 1 & 0.793 & & \\
$\begin{array}{l}\text { Corrección por continuidad } \\
\text { Razón de verosimilitudes }\end{array}$ & 0.000 & 1 & 1.000 & & \\
$\begin{array}{l}\text { Estadístico exacto de Fisher } \\
\text { Asociación lineal por lineal }\end{array}$ & 0.068 & 1 & 0.795 & & \\
N de casos válidos & 12 & 1 & 0.802 & & 0.682 \\
\hline
\end{tabular}

gl: grados de libertad; Sig.: significación.

A continuación, se analizan cada uno de los grupos en que se agruparon los servicios ecosistémicos que los pobladores señalaron recibir del humedal.

Servicios de aprovisionamiento. En la parte de aprovisionamiento, destaca la obtención de alimentos. Al respecto los pobladores afirman que se proveen de proteínas por medio del consumo de peces, animales silvestres y moluscos (aunque la última opción no es generalizada). Así mismo aprovechan animales domésticos y su producción.

Las abejas y avispas proveen de energía a las personas mediante la generación de miel que las personas cosechan de panales silvestres. Cabe mencionar que en la zona de estudio se presentan las condiciones naturales y climáticas para impulsar este tipo de actividades que podrían generar alimentos sanos, pero además contribuir a la mejora de ingresos económicos. El aprovisionamiento de alimentos se ha visto grandemente afectado por las constantes variaciones en el clima, sobre todo en las precipitaciones, lo que les ha limitado en los rendimientos productivos de los principales cultivos que componen la producción agrícola.

El agua dulce o de consumo en la laguna Moyúa podría clasificarse en dos grandes grupos: a) Uso consuntivo y b) Uso no consuntivo. En el primer caso se engloba el agua que al ser consumida cambia sus propiedades, es decir adquiere color, sabor y olor, además de su química. En el caso del uso no consuntivo, sobresale la navegación.

Los pobladores participantes del estudio señalaron cuatro principales fines; siendo estos: Agua de consumo humano, consumo animal, riego y navegación. Debido a las bajas precipitaciones en los dos últimos años, los manantiales se han secado. Lo que representa una grave situación para los habitantes de las comunidades, ya que estas carecen de servicio de agua potable, por lo que hacen uso de pozos, que según expresiones de los pobladores es "pesada, con olor a sarro", "llena de cal". Esta situación es debido a la concentración de sales minerales. Existe una estrecha relación entre la existencia de fuentes de agua dulce en cantidad, calidad y la posibilidad de establecer tanto como hacer producir en sistemas agrícolas, así como forestales. Los cultivos que componen los sistemas de producción de alimentos dependen exclusivamente de agua dulce. Según la FAO (2015), cerca del $60 \%$ de todo el volumen de las aguas extraídas de fuentes superficiales y subterráneas a nivel mundial es para uso en riego agrícola.

Mediante tabla de contingencia para las variables "Tiempo dedicado a acceder a agua" y "Género del participante", se determinó que quienes se ven mayormente afectados son las mujeres, debido a que son ellas y sus hijos menores, quienes se encargan de acarrear el agua para consumo y otros usos en el hogar. En las fincas que formaron parte del estudio, se observa la total dependencia de los sistemas de producción agropecuarios de la disponibilidad de agua de las fuentes superficiales (Las tres lagunas), en vista de las pocas precipitaciones, pero sobre todo en la forma errática en que ocurren estas en la zona. Las precipitaciones promedias en los últimos años han sido de 790 mm/año.

En el Cuadro 2, se muestran los resultados de análisis de contingencia.

Los sistemas ganaderos que poseen, además de proveerles de las proteínas necesarias (leche y carne), les sirven de fuentes de ingresos adicionales. El componente ganadero ha sido uno de los más afectados por la falta de agua 
Cuadro 2. Resultados de análisis de correlación a través de tabla de contingencia

\begin{tabular}{|c|c|c|c|c|c|}
\hline \multirow{2}{*}{\multicolumn{3}{|c|}{ Efecto de sequía sobre acceso a agua }} & \multicolumn{2}{|c|}{ Género del participante de estudio } & \multirow[t]{2}{*}{ Total } \\
\hline & & & Masculino & Femenino & \\
\hline \multirow{6}{*}{$\begin{array}{l}\text { Le lleva más tiempo } \\
\text { acceder a agua de consumo } \\
\text { si hay sequia }\end{array}$} & $\mathrm{Si}$ & Recuento & 3.0 & 5.0 & 8.0 \\
\hline & & $\%$ del total & 25.0 & 41.7 & 66.7 \\
\hline & No & Recuento & 3.0 & 0.0 & 3.0 \\
\hline & & $\%$ del total & 25.0 & 0.0 & 25.0 \\
\hline & No aplica & Recuento & 1.0 & 0.0 & 1.0 \\
\hline & & $\%$ del total & 8.3 & 0.0 & 8.3 \\
\hline \multirow{2}{*}{ Total } & & Recuento & 7.0 & 5.0 & 12.0 \\
\hline & & $\%$ del total & 58.3 & 41.7 & 100.0 \\
\hline
\end{tabular}

El MEM (2009) señala que el $60 \%$ de los hogares nicaragüenses utiliza la leña como principal combustible para las labores de preparación de alimentos, por ello en el país se consume anualmente cerca de tres millones de toneladas métricas. Con todo eso además de afectar con la deforestación, se provoca daños al aire, por la emisión de dulce, considerando que en las condiciones climáticas que prevalecen en la zona, una Unidad Animal (UAM) consume en promedio cerca de 70 litros de agua por día (aplicación de Formula de Murphy). Estudios a nivel mundial estiman que la ganadería es responsable del consumo de aproximadamente el 15\% del total de agua destinada a las actividades agrícolas. Otro componente importante desde donde se abastecen de proteínas, son los peces que extraen desde la laguna.

El 91.7\% de la población que formó parte de la investigación señalan que dependen de la provisión de proteínas desde la laguna. De esta importante valoración; el $83.3 \%$ señala como "Alto" su dependencia de la laguna para este servicio ecosistémico. El principal problema con las actividades pecuarias y los sistemas agrícolas, son las $\mathrm{CO} 2$ y otros gases perjudiciales (laprensa.com; agosto, 2013). Entre los principales problemas de salud se señalan: a) enfermedades respiratorias agudas, como la neumonía; b) enfermedades dérmicas; c) afecciones oculares, entre otros. Otro de los usos como aprovisionamiento, es el de tintes, siendo la principal fuente Calycophyllum candidissimun, el cual es utilizado para teñir cueros. Otro de los tintes, aunque no es utilizado por los productores es de Junglans olanchana. Los comunitarios señalaron que los ecosistemas les proveen de sustancias medicinales (Med), aromáticas (Aro), insecticidas (Ins), acaricidas (Aca) y repelentes (Ale). Las principales especies utilizadas se muestran en el Cuadro 3.

Cuadro 3. Especies de uso medicinal y otros usos suelos, falta de cobertura de suelos, el uso irracional de pesticidas y poco o escaso uso de pasturas mejoradas, así como el mal manejo de los sistemas ganaderos (falta de rotación de potreros, uso de cargas que superan la capacidad de los pastizales, entre otros). Esta problemática se podría resolver o reducir su impacto si se establecieran buenas prácticas agrícolas y pecuarias.

El $100 \%$ de las familias protagonistas del estudio manifestaron hacer uso de leña como principal fuente de energía para la cocción de alimentos. La leña utilizada es proveniente principalmente de especies como Acacia colliinsiisaff, Acacia dealbata, Albizia saman, Gliricidia sepium, entre otros. El 100\% de los habitantes que fueron entrevistados o participaron en el grupo focal señalaron que antes se extraían como promedio seis camionadas de leña cada semana. Eso es aproximadamente 60 metros cúbicos. Que asumiendo un rendimiento de entre 13-15 metros cúbicos/hectárea/año, representaba la deforestación de aproximadamente 205.71 hectáreas al año, equivalente a 292.14 manzanas. Esa extracción se ha minimizado significativamente, como producto de la sensibilización ambiental a través de la organización comunitaria.
El Gliricidia sepium se usa para controlar sarna de los perros y cerdos, pero también para controlar pulgas y jelepates. Otro uso extendido es quemar los termiteros para ahuyentar los zancudos y sabandijas que afectan tanto a las personas como los animales domésticos sobre todo en época lluviosa.

Las algas que colonizan las fuentes superficiales contienen ricas concentraciones de beta-caroteno y otros carotenoides ampliamente empleados en la industria farmacéutica. Su uso aun es poco o casi nulo en las comunidades estudiadas. El mayor uso ha sido de Jacinto de agua y lechuga de agua para la producción de abonos orgánicos. El uso como abono orgánico se ha mencionado por parte de pobladores como una iniciativa para reducir la proliferación de estas 
plantas acuáticas y darle un uso agrícola. Esta iniciativa fue promovida por la UNAN Managua a través de sus proyectos en dicha zona. Como recursos genéticos se han identificado una gran cantidad de especies de aves, de flora (terrestre y acuática). En estudio de caso publicado por Montenegro (2012, p.13), se señala que en el ecosistema se reporta la existencia de:

"unas 215 especies de organismos en total. Se ha observado 57 especies de aves, tanto migratorias como locales. La flora terrestre circundante consiste en 41 especies, y la acuática o hidrófita en 25 . Se ha identificado a 5 especies de peces, 26 mamíferos, y 6 reptiles. En las aguas de Moyúa, se ha identificado a 25 especies de algas (fitoplancton), 11 especies del zooplancton y a 19 especies del zoobentos".

Toda esta riqueza biológica en tan poco espacio lo hace ser un sitio prioritario para su protección y el incentivo de sistemas más integrales de producción agropecuaria y forestal.

\section{Servicio ecosistémico de regulación}

Clima. El 100\% de los pobladores señalan que la existencia de los bosques y la laguna les ayuda a mantener un clima agradable, sobre todo en esta zona donde la temperatura es bastante alta. La existencia de árboles, además de proveerles de frutas, leña, medicinas, entre otros, les sirve para regular temperatura, el cual es provechoso sobre todo para el ganado que pasta en los potreros o que se encuentran encerrados en los corrales. Un beneficio más que se le atribuye a los bosques y suelos, es la retención de humedad, la captura y secuestro del $\mathrm{CO}_{2}$ y la producción de agua y oxígeno.

Erosión. La cobertura tanto aérea como de suelos es de vital importancia por su papel en la reducción de las tasas de erosión, lo que constituye un servicio ecosistémico de regulación.

Enfermedades y plagas. Los productores y sus familias han observado que, debido a los árboles, hay más pájaros y otros "animales" que se alimentan de zancudos (Culicidae) y otras plagas, que afectan a las personas, los cultivos y a su ganado. La existencia de especies arbustivas, malezas y árboles, también permite que estos sirvan como barreras para evitar el ataque de plagas y enfermedades a los cultivos. Los suelos cuando están cubiertos por vegetación o por hojarasca son capaces de retener humedad, con lo cual se da la posibilidad de albergar insectos y otro tipo de artrópodos que son controladores de plagas y la transmisión de enfermedades tanto a las personas como a los animales domésticos.
Polinización. Este servicio ecosistémico es poco reconocido por los productores, quienes ven a los insectos como dañinos para los cultivos o bien en el caso de las abejas (Aphis mellifera) solo para producir miel y cera. El cuido y protección de los insectos tiene una importancia estratégica. La polinización efectuada por los insectos y específicamente por abejas con o sin aguijón, contribuye a mantener e incluso elevar los rendimientos productivos y la calidad de los principales cultivos que se establecen en la zona.

Saneamiento y agua. Este tipo de servicio ecosistémico fue identificado más para los suelos, los que retienen una gran cantidad de pesticidas y herbicidas, así se evita que contaminen las aguas superficiales y subterráneas, desde donde se abastecen para el consumo humano y de sus animales. Por otro lado, se hace mención de las plantas acuáticas que extraen los nutrientes en el cuerpo de agua y que luego estas plantas pueden ser aprovechadas para la producción de abonos orgánicos. En hecho de los arrastres de sedimentos hacia la laguna de Moyúa ha creado serios problemas por la eutrofización, ocasionando la polución de plantas acuáticas como Eichornia crassipes, Pistias tratiotes y Salvinia natans, que hace que se reduzca el espejo de agua, además de crear un ambiente carente de oxígeno y luz; por lo tanto se afecte las poblaciones de peces y moluscos, que son la fuente de alimento para muchas de las especies de aves migratorias y nativas, pero además se afecte el suministro de alimentos e ingresos a las personas que dependen de los peces o el turismo.

\section{Servicios ecosistémicos culturales}

Espiritual y religioso. Los servicios ecosistémicos "Espiritual y Religioso" incluye la inspiración estética, la identidad cultural, el sentimiento de apego al terruño, así como la experiencia espiritual relacionada con el entorno natural. Normalmente, en este grupo se incluyen también las oportunidades para el turismo y las actividades recreativas.

Los servicios culturales están estrechamente interconectados, a menudo están relacionados con los servicios de abastecimiento y de regulación: la pesca en pequeña escala no solo tiene que ver con los alimentos y los ingresos, sino también con el modo de vida de los pescadores. Que en el caso de Moyúa se han dedicado a otras actividades productivas, dejando la pesca solo para proveerse de proteínas, al no poder adquirir otras fuentes como la carne de res, de cerdo o pollo.

En muchos casos, los servicios culturales figuran entre los valores más importantes que las personas asocian con la naturaleza; es por ello fundamental comprenderlos. El humedal reviste una gran importancia en el aspecto cultural, por su importante valor en lo espiritual y religioso. La mayoría de los visitantes de la laguna llegan para sentirse 
bien y poder tener un momento de tranquilidad que le ayude a mejorar su espiritualidad y para quienes creen en Dios, la posibilidad de agradecer la existencia personal y de sitios como este en una zona tan seca. En el sitio Ramsar Moyúa, se tiene la presencia de ruinas indígenas que posiblemente se utilizaban para cuestiones religiosas. Este tipo de edificaciones se encuentran sobre todo en la zona de Moyúa, en el sector 1 (terrenos de Don Narciso Moreno) y en el sector conocido como la isla (donde no hay acceso a particulares). En el Sector 1, el Señor Narciso Moreno, encontró artefactos utilizados por los indígenas.

Recreativo, turístico y estético. La misma localización del complejo lagunar lo convierte en un sitio de interés desde el punto de vista turístico y recreativo. La zona es una de las más secas del país y el hecho de tener un cuerpo de agua dulce como ese invita a quienes le ven a conocerle más a profundidad, dando la oportunidad a los habitantes de la zona, de brindar servicios de turismo, sobre todo del tipo rural comunitario. En el cuadro 4, se logra observar la estrecha correspondencia entre la valoración que los pobladores le dan a la laguna y la actividad turística como generadora de ingresos a las familias que habitan en los alrededores. El 83.3\% de los participantes del estudio consideran como "Alto" el valor que tiene la laguna para ellos. Al indagar sobre si el turismo le genera ingresos económicos, el $66.7 \%$ de los involucrados en el estudio, respondió afirmativamente; con lo que se demuestra que el turismo depende de la existencia y buen estado de la laguna.

Para fortalecer este tipo de oportunidades (turismo), el CIRA-UNAN Managua ha ejecutado importantes acciones con ayuda del PNUD y GWP; equipándoles de lanchas con motor y acondicionando las viviendas. En este esfuerzo se ha unido en los últimos tres años la FAREM Matagalpa a través de la carrera de Ingeniería Agronómica, con la realización de estudios y acciones para mejorar la parte productiva y ambiental.

La existencia del espejo de agua en un paraje tan seco, hace del sitio un oasis para las aves migratorias y las aves nativas. En el sitio se pueden avistar aves como la cerceta aliazul (Anas discors), cerceta castaña (Anas cyanoptera), pato cuchara (Anas clypeata), la garza blanca (Casmerodios albus), garza morena (Florida caerulea), garza africana del ganado (Bubulcus ibis) y el piche (Dendrocygna autumnalis). Estas especies constituyen un atractivo para los visitantes, que ha sido aprovechado por los habitantes cuando la laguna se encuentra llena. Acerca de la existencia del espejo de agua y la actividad turística motivada por el avistamiento de aves migratorias y nativas; el $91.7 \%$ manifiesta que las aves constituyen uno de los atractivos turísticos, pero que estas dependen de la existencia del espejo de agua. En las estaciones cuando la laguna se ha secado, las aves no llegan a la laguna, pero por otro lado no hay servicio de las lanchas, por dificultarse la navegación, debido al bajo nivel de las aguas.

Educativo. En el sitio Ramsar del complejo lagunar Moyúa, se han desarrollado una serie de investigaciones tanto a nivel de pregrado como de postgrado, las más recientes son precisamente el documento presente y cinco tesis a nivel de postgrado (dos de maestrías y tres de doctorado). Además, se han desarrollado una serie de acciones entre estudiantes de universidades (UNAN Managua, UPONIC, Universidad de Agricultura de OlanchoHonduras, entre otras) y productores de las comunidades del sitio Ramsar. La Laguna de Moyúa posee una serie de atractivos que la hacen propicia para la realización de estudios tanto en aspectos sociales, antropológicos, económicos, ambientales y en otras áreas del conocimiento.

Identidad de sitio y herencia cultural. En el sitio se encuentran vestigios de las poblaciones indígenas que habitaron estos territorios, asimismo se han encontrado artefactos utilizados en actividades de preparación de alimentos y de labranza de suelo, utilizados por las poblaciones indígenas. La existencia de este tipo de infraestructura y artefactos, hace que el sitio tenga un mejor atractivo y hace que las personas se sientan orgullosas de su origen. Pero además se abre la oportunidad de que se entiendan más en la conservación del sitio y de su capital natural y cultural.

\section{Servicio ecosistémico de soporte}

Formación de suelos y ciclaje de nutrientes. Las condiciones climáticas del sitio hacen posible un proceso más acelerado de formación de suelos (debido a microclima), pero además por la producción de hojarasca al darse las condiciones propicias para el crecimiento de vegetación tanto arbustiva como arbórea. La hojarasca provee las condiciones y alimentos para la reproducción y funcionamiento de toda una cadena compuesta por artrópodos, mamíferos, aves, reptiles, entre otros, que al final contribuyen a la formación y mejoramiento de los suelos de los 
alrededores de las lagunas, que componen el sitio Ramsar. El problema que se ha observado sobre todo en años anteriores a la intervención del CIRA y la carrera de agronomía de la FAREM Matagalpa, es que las personas queman los rastrojos de cosecha o en vez de chapiar los potreros le "pegan fuego", con lo cual dañan los suelos, pero además provocan emisiones de $\mathrm{CO}_{2}$, matan a los animales silvestres y perjudican el hábitat de las especies silvestres.

Al indagar entre los involucrados en el estudio acerca del efecto de las quemas agrícolas sobre el suelo, el $75 \%$ señaló que sus suelos se ven dañados al efectuarse quemas agrícolas. Al profundizar porque realizan estas prácticas si saben que son perjudiciales; manifestaron que se debe a la falta de recursos para contratar mano de obra y que aún falta conocimiento acerca del papel de los rastrojos en mantener la humedad de suelos, mejorar la diversidad en los suelos y reducir la pérdida de suelos y nutrientes.

Cuadro 5. Efecto de las quemas agrícolas sobre las parcelas (suelos)

\begin{tabular}{lccccc}
\hline \multicolumn{1}{c}{ Indicador } & Valor & gl & $\begin{array}{c}\text { Sig. asintótica } \\
\text { (bilateral) }\end{array}$ & $\begin{array}{c}\text { Sig. exacta } \\
\text { (bilateral) }\end{array}$ & $\begin{array}{c}\text { Sig. exacta } \\
\text { (unilateral) }\end{array}$ \\
\hline $\begin{array}{l}\text { Chi-cuadrado de Pearson } \\
\text { Corrección por continuidad }\end{array}$ & $0.114^{\mathrm{a}}$ & 1 & 0.735 & & \\
$\begin{array}{l}\text { Razón de verosimilitudes } \\
\text { Estadístico exacto de Fisher }\end{array}$ & 0.116 & 1 & 0.733 & & \\
$\begin{array}{l}\text { Asociación lineal por lineal } \\
\text { N de casos válidos }\end{array}$ & $\begin{array}{c}0.105 \\
12\end{array}$ & 1 & 0.746 & 1.000 & 0.636 \\
\hline
\end{tabular}

a. 3 casillas $(75.0 \%)$ tienen una frecuencia esperada inferior a 5. La frecuencia mínima esperada es 1.25 .

b. Calculado sólo para una tabla de $2 \times 2$. gl: grados de libertad; Sig.: significación.

El relieve variado facilita el arrastre de las rocas, que al irse desfragmentado facilitan la formación de suelos, pero además se da la colonización de rocas por musgos, líquenes, plantas superiores e insectos que promueven la meteorización de estas. A sí mismo, es responsable de procesos de desgaste de suelos debido a los procesos erosivos hídricos y eólicos. Los arrastres de suelo de las partes altas y medias son depositados en las partes bajas hacen que estas zonas sean más productivas, por su misma fertilidad.

Valoración de los servicios ecosistémicos. La valoración de los servicios ecosistémicos prestados por el sitio Ramsar de complejo lagunar de Moyúa, representó grandes retos, debido a la falta de una guía de fácil aplicación para que se lograra la participación de los comunitarios por lo que se considera que debido a ello los bienes y servicios ecosistémicos no son valorados adecuadamente. De hecho, su estudio a nivel nacional es escaso o poco preciso, por lo cual la protección de los bosques, humedales y otros tipos de ecosistemas es visto como poco rentable.
Bustamante y Ochoa (2014, p.28) en un estudio publicado por WWF Perú y el SNV, señalan que "la visión económica neoclásica, la valoración es una medida de la capacidad de los ecosistemas para satisfacer necesidades esenciales a la vida". La misma fuente señala que los servicios que el ecosistema presta a la humanidad se pueden valorar desde distintas perspectivas. Una de ellas está centrada en el "ser humano y el valor que este le asigna a los bienes y servicios del ecosistema”. Otra posibilidad es valorar el ecosistema en base a sus características propias. Esto conlleva a la "valoración cultural, espiritual y religiosa". En el caso del presente estudio se utilizó una combinación de ambos métodos, ya que los aportes del ecosistema pueden ser tangibles como intangibles y ambos están interrelacionados.

Para poder valorar los servicios ecosistémicos, de previo se habían identificado los principales servicios que desde la percepción de los pobladores se reciben del humedal. Los humedales y los demás sistemas naturales, tienen una gran importancia social y económica. Instituciones como la FAO (2015, p.23) señalan que los humedales junto a suelos, bosques y ríos, deben ser protegidos y restaurados por su importante papel en "aumentar la disponibilidad de agua de buena calidad". En el caso de la Laguna de Moyúa, los ingresos por actividades de turismo ecológico contribuyen a la economía local. Estos ingresos son debido a la prestación de servicios de traslado en lancha, la venta de productos agropecuarios a los visitantes, la venta de alimentos ya preparados, etc.

Se hace necesario contar con metodologías que permitan comprender mejor la valoración de los servicios ecosistémicos, de esa forma poder impulsar su aplicación en las políticas públicas y facilitar la toma de decisiones financieras, proyectos sociales o las mismas inversiones en las zonas que prestan servicios ecosistémicos, como es el caso de Moyúa.

\section{CONCLUSIONES}

Se concluye que los pobladores que formaron parte de la investigación, están conscientes de la importancia de los servicios ecosistémicos que les proveen los componentes de los ecosistemas presentes en el sitio Ramsar y su impacto en el bienestar humano.

Existe dificultad para poder cuantificar monetariamente los servicios ecosistémicos que se perciben de parte de los diferentes componentes de los ecosistemas presentes en el humedal. 
Existe compromiso de parte de los pobladores para conservar y restaurar suelos como uno de los principales componentes del capital natural vinculado al bienestar de las familias.

Existe poca presencia y apoyo de parte de la municipalidad y las instituciones del Estado en la preservación y gestión del humedal a pesar de ser un sitio Ramsar.

\section{RECOMENDACIONES}

Impulsar estrategias para: manejo integrado de recursos hídricos, suelos y bosques; promover la preservación de los vestigios de civilización indígena, para lo cual se puede acondicionar área en la oficina de información a visitantes de la laguna de Moyúa; regular la pesca y la caza; realizar obras de conservación de suelos tales como: curvas a nivel, barreras vivas, barreras muertas, terrazas, incorporación de rastrojos al suelo, diques de contención, entre otras; realizar rotación de cultivos para evitar la proliferación de plagas y enfermedades; para los productores que poseen grandes áreas de producción, se recomienda dejar barbechos a los suelos; implementar cosecha de agua; en el caso de la conservación de bosques es recomendable establecer cultivos en asocio con el bosque, y en caso de la ganadería, se puede fomentar el sistema silvopastoril.

\section{AGRADECIMIENTOS}

Para la realización de la investigación, se contó con el valioso apoyo financiero de parte del Fondo de Proyectos de Investigación (FPI) de la UNAN Managua, al cual deseamos agradecer y dedicar la presente publicación. Agradecemos la colaboración de las familias que formaron parte fundamental en la investigación. Especial agradecimiento a los señores: Narciso Moreno Orellana (q.e.p.d.) y a su esposa Emelda, a Jorge Moreno Vásquez y la Señora Flora Orozco, como líderes comunitarios. Nuestros agradecimientos al CIRAUNAN Managua, a la maestra Thelma Salvatierra y al maestro Salvador Montenegro por su valiosa colaboración brindada.

\section{REFERENCIAS BIBLIOGRÁFICAS}

Aguilera, A. (27 de febrero, 2010). Moyúa espera por vos. La Prensa. https://www.elnuevodiario.com.ni/especiales/69241-moyuaespera-vos/

Balzarini, M., Di Rienzo, J., Tablada, M., González, L., Bruno, C., Córdoba, M., Robledo, W. y Casanoves, F. (2015). Estadistica y Biometría: Ilustraciones del Uso de InfoStat en Problemas de Agronomía. cuenca del río Otún-Pereira, Colombia. CATIE, Turrialba, Costa Rica.

Bortz, J., Lienert, G., y Boehnke, K. (1990). Verteilungsfreie Methoden in der Biostatistik. (Métodos libres de distribución en la bioestadística) Springer, Berlin.

Bustamante, A., y Ochoa, C. (2014). Guía práctica para la valoración de servicios ecosistémicos. WWF, Perú. 45 p.

FAO (Organización de las Naciones Unidas para la Alimentación y la Agricultura). (2015). Construyendo una visión común para la agricultura y alimentación sostenibles. Principios y enfoques. Roma. ISBN 978-92-5-308472-2, 55 p.

MEA (2005). Ecosystems and human well-being: Policy responses. Washington, DC, USA. Island Press. $621 \mathrm{p.}$

MEM (Ministerio de Energía y Minas). (2009). Estrategia Nacional de Leña y Carbón Vegetal de Nicaragua. Managua, 47 p.

Montenegro, S. (2012). Experiencias para la adaptación y reducción de la vulnerabilidad al cambio climático en el sector hídrico: Caso Humedales de Moyúa, Playitas y Tecomapa, Nicaragua. Global Water Partnership.

Santos, T., y Tellería, J. (2006). Pérdida y fragmentación del hábitat: efecto sobre la conservación de las especies. Revista Ecosistemas, 15. AAET. 10 p. 